\title{
A Case Report of Life-Threatening Succinylcholine-Induced Anaphylaxis: Diagnostic Difficulty and Management
}

\author{
M Ben Ali ${ }^{1 *}$, K. Maamri ${ }^{2}$, F. Ben Salem ${ }^{1}$, M Dermoul $^{2}$, M Gahbiche ${ }^{2}$ \\ ${ }^{I}$ Anesthesia-Resuscitation Departement Hospital Fattouma Bourguiba Monastir-TUNISIA \\ ${ }^{2}$ Neurosurgery Departement Hospital Fattouma Bourguiba Monastir-TUNISIA
}

*Corresponding Author: M Ben Ali, Anesthesia-Resuscitation departement hospital Fattouma Bourguiba Monastir-TUNISIA

\section{INTRODUCTION}

Perioperative anaphylactic reactions always remain a major concern for any Anesthesiologist (1). The majority of reactions appear immediately in minutes following the injection of these products (2). Succinylcholine is the primary provider of anaphylactic shock in anesthesia, given the frequent use of this molecule for rapid sequence intubation, defined, as the simultaneous administration of a sedative and a neuromuscular blocking agent the succinylcholine (suxamethonium), difficult intubation according to SFAR recommendations and seismotherapy to avoid the side effects of convulsions caused (3). Anaphylaxis is not a common side effect of these drugs, they rarely happen. That is why it is difficult to determine the causality of succinylcholine in such adverse reaction. Preventing, diagnostic and treating and anaphylactic reaction in the operating room is a challenge for the intensive care anesthesiologist. We report a real clinical case of anaphylactic shock to succinylcholine, which occurred in the neurosurgery unit of the Monastir Hospital.

\section{OBSERVATION}

This case concerns a 7-month-old infant who have to undergo a ventriculoperitoneal shunt surgery. This patient has been operated 3 times previously but without the use of succinylcholine. The anesthesia was induced with propofol and suxamethonium. Five minutes after the induction, the systolic blood pressure dropped suddenly to $50 \mathrm{mmHg}$, the heart rate decreased to 45 beats per minute and the oxygen saturation dropped to $55 \%$. A small degree of bronchospasm developed but there was not any kind of skin reaction. We thought of an allergic reaction. Cardiopulmonary resuscitation has been initiated. Within $10 \mathrm{~min}$, all vital signs normalized, mydriasis regressed after 3 hours. The operation was cancelled. The infant was kept intubated, ventilated and sedated in the operating room during 6 hours because there was no extra place available in the pediatric resuscitation unit. Six hours after, the patient was extubated and transferred to the neurosurgery department. Thereafter the pharmacovigilance concluded that the role of suxamethonium in this case cannot be missed and it is absolutely contraindicated to use it for this baby. Skin tests will be performed later. The patient was operated later without incident, he undergo a ventricul operitoneal shunt surgery without the use of succinylcholine.

\section{Comment}

This case series documents the acuteness, suddenness and severity of cardiovascular events in succinylcholine-related anaphylaxis and demonstrates the difficulty of diagnosing anaphylaxis, which led to frequent sub-optimal and/or in appropriate management at the time of anaesthetic induction $(5,6)$.

\section{CONClusion}

Anaphylaxis during anesthesia is a rare phenomenon but might have life threatening consequences if it is not promptly diagnosed and treated at time. The incidence of anaphylaxis to succinylcholine was about 1/2,000 (4). Close collaboration between the allergologist and the anesthesiologist is a key issue when investigating anaphylactic reactions.

\section{REFERENCES}

[1] Death from anaphylactic shock in curares: analysis of risk factors and therapeutic management. Thesis of doctor in medecine sustained july 12, 2013. 
[2] PM Mertes et al. Anaphylactic shock. SFAR 2014.

[3] Communique concerning the modification of the SPC for Celocurine. SFAR press releases. Posted on February 8, 2018

[4] General anesthetics and intraoperative anaphylaxis, the hiden side of the 'block' General anesthetics and perioperative anaphylaxis: The side of the operatingroom; Master thesis in medecine. Departement of immunology and allergy, Lausanne on 15. 12.2016.

[5] Mertes PM, De Blay F, Dong S, Allergic risk in aneshesia ; immunology/ EmergenciesResucitation Anesthesia 2012 ; 42 : 269-279.

[6] Immediate per-anesthesic hypertensitivity reactions to $\mathrm{CHU}$ de potnmmmmmmmmmm ,k,'nnnnnnnnnn/u4iers in 2012 and 2013.

Citation: M Ben Ali, K. Maamri, F. Ben Salem, M Dermoul, M Gahbiche, " A Case Report of LifeThreatening Succinylcholine-Induced Anaphylaxis: Diagnostic Difficulty and Management. ARC Journal of Anesthesiology. 2020; 5(2): 20-21. DOI: doi.org/ 10.20431/2455-9792.0502004.

Copyright: () 2020 Authors. This is an open-access article distributed under the terms of the Creative Commons Attribution License, which permits unrestricted use, distribution, and reproduction in any medium, provided the original author and source are credited. 